\title{
Bioactive Equivalence of Combinatorial Components Identified in Screening of an Herbal Medicine
}

Peng Liu • Hua Yang • Fang Long • Hai-Ping Hao • Xiaojun Xu • Ying Liu • Xiao-Wei Shi • Dan-Dan Zhang • Hao-Chuan Zheng • Qian-Ying Wen • Wen-Wen Li • Hui Ji • Xi-Juan Jiang • Bo-Li Zhang • Lian-Wen Qi • Ping Li

Received: 21 October 2013 / Accepted: 31 December 2013 / Published online: 19 February 2014

(C) The Author(s) 2014. This article is published with open access at Springerlink.com

\begin{abstract}
Purpose To identify bioactive equivalent combinatorial components (BECCs) in herbal medicines. The exact composition of effective components in herbal medicines is often elusive due to the lack of adequate screening methodology. Herein, we propose a hypothesis that BECCs accounting for the whole efficacy of original herbal medicines could be discovered from a complex mixture of constituents.
\end{abstract}

Methods We developed a bioactive equivalence oriented feedback screening method and applied it to discover the BECCs from an herbal preparation Cardiotonic Pill (CP). The operations include chemical profiling of CP, followed by an iterative loop of determining, collecting and evaluating candidate BECCs.

Results A combination of 18 compounds was identified as BECCs from CP, which accounts for $15.0 \%(\mathrm{w} / \mathrm{w})$ of original CP. We have demonstrated that the BECCs were as effective as $\mathrm{CP}$ in cell models and in a rat model of myocardial infarction.

Conclusions This work answers the key question of which are real bioactive components for CP that have been used in clinic for many years, and provides a promising approach for discovering BECCs from herbal medicines. More importantly, the BECCs could be extended to improve quality control of herbal products and inspire an herbal medicines based discovery of combinatorial therapeutics.

Peng Liu, Hua Yang and Fang Long contributed equally to this work.

Electronic supplementary material The online version of this article (doi: | 0. I007/s I 1095-0 13- | 283- I) contains supplementary material, which is available to authorized users.

P. Liu $\cdot$ H. Yang $\cdot$ F. Long $\cdot$ H.-P. Hao $\cdot$ X. Xu $\cdot$ Y. Liu $\cdot$ X.-W. Shi $\cdot$

D.-D. Zhang $\cdot$ H.-C. Zheng $\cdot$ Q.-Y. Wen $\cdot$ W.-W. Li $\cdot H . ~ J i \cdot$

L.-W. Qi $(\bowtie) \cdot$ P. Li $(\bowtie)$

State Key Laboratory of Natural Medicines

China Pharmaceutical University, Nanjing 210009, China

e-mail: liping2004@I26.com

e-mail: fleude@।26.com

X.-J. Jiang • B.-L. Zhang

Tianjin University of Traditional Chinese Medicine, Tianjin 300 I 93, China
KEY WORDS bioactive equivalence · Cardiotonic Pill . combinatorial components screening · herbal medicines

\section{ABBREVIATIONS}

BECCs Bioactive equivalent combinatorial components

CCK-8 Cell counting kit-8

CK-MB Creatine kinase-MB

CP Cardiotonic Pill

HUVEC Human umbilical vein endothelial cell

IL-6 Interleukin-6

LAD Left anterior descending

$\mathrm{LDH} \quad$ Lactate dehydrogenase

LPS Lipopolysaccharide

LV Left ventricular

LVEDP Left ventricular end diastolic pressure

LVSP Left ventricular systolic pressure

MI Myocardial infarction

MRCs Mixture of reference compounds

Ox-LDL Oxidized low density lipoprotein

PGE2 Prostaglandin E2

PLS-DA Partial least square-discriminant analysis

ROS Reactive oxygen species

SD Standard deviation

SI Simulated ischemia

TNF- $\alpha$ Tumor necrosis factor- $\alpha$

TTC Triphenyltetrazolium chloride

\section{INTRODUCTION}

Herbal medicines have played an important role in health maintenance and disease treatment for thousands of years $(1,2)$. Accompanying with hot discussions on developing multidrug therapy for multi-gene diseases, herbal medicines are receiving increasing attention worldwide because they have long been postulated as multicomponent therapeutics in clinical 
practice (3-7). Nevertheless, the exact composition of effective components is often elusive due to the lack of adequate screening methodology. A variety of research efforts in recent decades have been focused on isolating and identifying single effective constituents from herbal medicines. However, they disregarded the combinatorial role and integrative therapeutic effects of multiple active compounds $(8,9)$. Generally, the therapeutic efficacy of herbal medicines is achieved by combinatorial components rather than single compound. Hence, it is incumbent upon researchers to elucidate the exact combinatorial composition of effective components in herbal medicines, if any, accounting for of the holistic efficacy of herbal medicines.

In this work, we propose a hypothesis that bioactive equivalent combinatorial components (BECGs) accounting for the whole efficacy of original herbal medicines could be discovered from a complex mixture of constituents. To explore BECGs from herbal medicines, we have developed and described herein a bioactive equivalence oriented feedback screening method, and applied it to discover the BECCs from an herbal preparation Cardiotonic Pill (CP, also known as the Compound Danshen Dripping Pill). CP is a Chinese herbal medicinal preparation in which Salviae Miltiorrhizae Radix and other two Chinese medicines, namely Notoginseng Radix and borneolum, were combined in a fixed ratio using modern techniques of pharmaceutical preparation. CP has been widely applied clinically in China to improve cardiac function and coronary circulation for the therapy of angina pectoris $(10,11)$ and has recently been approved to enter Phase III clinical trials by the FDA (ClinicalTrials.gov Identifier: NCT01659580). Herein, we have identified a combination of 18 compounds as BECGs out of 36 identified components and other unidentified minor components, which were as effective as CP in cell models and in a rat model of myocardial infarction (MI). The content of BECGs accounts for $15.0 \%(\mathrm{w} / \mathrm{w})$ of original CP, and could be considered as the "defined labeled amount" of active component combination for CP.

This work offers evidence-based data to answer the key question of which are real bioactive components for Cardiotonic Pill that have been used in clinic for many years. While the nature of this study refers specifically to Cardiotonic Pill, we suggest that this screening method is promising for detecting BECGs for other herbal medicines, and more importantly, to improve quality control of herbal medicines and inspire an herbal medicines based discovery of combinatorial therapeutics.

\section{METHODS}

\section{Chemical Profiling of CP}

To select candidate BECGs from CP, we initially profiled the chemical constituents of CP. Three batches of CP (Tasly
Pharmaceutical Co., Ltd., Lot: 100824, 110419, 110510) were obtained from local drug stores (Nanjing, China). Reference compounds were obtained from the National Institute for The Control of Pharmaceutical and Biological Products (Beijing, China) or previously preserved in our laboratory. The purity of each reference compound was determined to be higher than 98\%. For HPLC-UV/MS analysis, CP (15 mg, without addition of excipients) was extracted using $75 \%$ (v/v) ethanol $(3 \mathrm{ml}$ ) for $30 \mathrm{~min}$ by ultrasonication and subsequently centrifuged at $13,000 \mathrm{rpm}$ for $10 \mathrm{~min}$. The supernatant was analyzed on an Agilent 1100 series HPLC system (Agilent, USA). The separation was performed on an Agilent Zorbax SB-C18 column $(250 \times 4.6 \mathrm{~mm}, 5 \mu \mathrm{m})$ using the $0.02 \%$ formic acid in water $(\mathrm{A})$ and $0.02 \%$ formic acid in ACN (B). The gradient elution program was $10-22 \% \mathrm{~B}$ at 0 $10 \mathrm{~min}, 22-23 \% \mathrm{~B}$ at $10-18 \mathrm{~min}, 23-24 \% \mathrm{~B}$ at $18-23 \mathrm{~min}$, $24-27 \%$ B at 23-33 min, 27-33\% B at 33-40 min, 33-41\% B at $40-52 \mathrm{~min}, 41-70 \% \mathrm{~B}$ at $52-54 \mathrm{~min}, 70-72 \% \mathrm{~B}$ at $54-$ $62 \mathrm{~min}, 72-100 \% \mathrm{~B}$ at $62-70 \mathrm{~min}, 100 \% \mathrm{~B}$ at $70-77 \mathrm{~min}$. The flow rate was kept at $0.5 \mathrm{ml} / \mathrm{min}$ at $30^{\circ} \mathrm{C}$. For segmental monitoring (12) based on UV, different detection wavelengths were performed for different periods of time: $203 \mathrm{~nm}$ for 0 $57 \mathrm{~min}$; $281 \mathrm{~nm}$ for $57-77 \mathrm{~min}$. MS spectra were acquired on a 6530 Q-TOF mass spectrometer (Agilent, USA) equipped with an electrospray ionization (ESI) interface. The operating parameters were as follows: drying gas $\left(\mathrm{N}_{2}\right)$ flow rate, $10.0 \mathrm{~L} /$ min; drying gas temperature, $320^{\circ} \mathrm{C}$; nebulizer, 45 psig; capillary, 3,500 V; skimmer, $65 \mathrm{~V}$; OCT RFV, $750 \mathrm{~V}$; and fragmentor voltage $120 \mathrm{~V}$. For MS/MS experiments, the collision energy was adjusted from 15 to $70 \mathrm{~V}$ to optimize signals and obtain maximal structural information from the ions of interest. The sample was analyzed in both positive and negative modes. The mass range was set at m/z 100-3,000.

\section{Trapping and Preparing Candidate BECCs}

To obtain the combination of candidate BECGs, we applied the real-time components trapping and combining system for sample preparation (Figure S1, Supplementary Material). A preparative Agilent 1100 series HPLC system was coupled to a LEAP Shell fraction collection system (LEAP Technologies, USA). The fraction collection system was composed of an autosampler (HTC PAL MXY 04-01A), a system control software (LEAP Shell 3) and a fraction collector (HTG PAL MXY 013-02A). The HPLG separation was performed on an Agilent Zorbax SB-C18 semi-preparative column $(250 \times 9.4 \mathrm{~mm}, 5 \mu \mathrm{m})$ with a flow rate of $2 \mathrm{ml} / \mathrm{min}$, and other HPLC conditions were the same as those in chemical profiling of CP.

After the injection of $20 \mu \mathrm{l} \mathrm{CP}$ at the concentration of $100 \mathrm{mg} / \mathrm{ml}$ in $75 \%$ (v/v) ethanol, candidate BECGs were prepared using the real-time components trapping and combining system (Figure S1, Supplementary Material) according 
to the collection program as following: P2 at 648-690 s, P3 at 996-1,050 s, P5 at 1,308-1,380 s, P6 at 1,386-1,446 s, P7 at 1,548-1,620 s, P8-P9 at 1,866-1,992 s, P10 at 2,001-2,052 s, S3 at 2,058-2,130 s, P11 at 2,256-2,382 s, P12 at 2,640$2,772 \mathrm{~s}, \mathrm{~S} 5$ at 3,060-3,102 s, S9 at 3,312-3,348 s, S12 at 3,402-3,438 s, T3 at 4,110-4,146 s, T4-T5 at 4,356-4,440 s, $\mathrm{T} 7$ at 4,560-4,602 s. Candidate BEGGs were collected at position II while the remaining part was collected at position I. Subsequently, the solvent was removed using a speedvac evaporator (Genevac EZ-2 plus, Genevac Technologies, UK). The samples were reconstituted at a concentration as in original CP for method validation and bioactivity assay.

\section{Bioactivity Assays In Vitro}

To assess the bioactive equivalence, we evaluated the bioactivities of candidate BECCs and the original CP simultaneously. Three cell models related to angina pectoris were used $(13,14)$, including oxidized low density lipoprotein (Ox-LDL)mediated human umbilical vein endothelial cell (HUVEG) injury (15), lipopolysaccharide (LPS)-stimulated RAW 264.7 macrophage inflammation (16), and simulated ischemia (SI)induced H9c2 cardiomyocytes injury (17). If the 90\% confidence interval (CI) of relative efficacy compared to original CP fell within the range of $70-143 \%$ (18), the candidate BECGs were considered to be a bioactive equivalent with original CP.

HUVEC (EA.hy926), mouse macrophage (RAW 264.7) and rat cardiomyoblast $(\mathrm{H} 9 \mathrm{c} 2)$ cell lines were purchased from American Type Culture Collection (ATCG, USA). Cells were grown at $37^{\circ} \mathrm{C}$ under a humidified atmosphere with $5 \%$ (v/v) $\mathrm{CO}_{2}$ in accordance to optimal media and growth conditions specified by ATCC.

To assess the effect of candidate BECGs on HUVEGs, we pretreated cells with various samples (corresponding concentrations in $0.4 \mathrm{mg} / \mathrm{ml}$ Cardiotonic Pill) or Trolox $(100 \mu \mathrm{M}$, as a positive control) for $12 \mathrm{~h}$. After preincubation, supernatant was removed and cells were subjected to Ox-LDL $(70 \mu \mathrm{g} / \mathrm{ml}$, XieSheng Biotechnology, China) for $24 \mathrm{~h}$ except the vehicle control group. Cell viability was determined by cell counting kit-8 (CCK-8, Dojindo Molecular Technologies Inc., Japan). Supernatant was collected for lactate dehydrogenase (LDH) assay (Jiancheng Bioengineering Institute, China) according to the manufacturer's instructions. ROS production in HUVECs was determined by a fluorometric assay using DCFH-DA (Beyotime, China) as previously reported (19).

To assess the anti-inflammatory effect of candidate BECGs, the macrophages were pretreated for $2 \mathrm{~h}$ with various samples (corresponding concentrations in $0.5 \mathrm{mg} / \mathrm{ml}$ Cardiotonic Pill) or luteolin $(20 \mu \mathrm{M}$, as a positive control $)$ and then stimulated for $20 \mathrm{~h}$ with LPS $(1 \mu \mathrm{g} / \mathrm{ml})$. The NO production was determined as nitrite concentration in the culture medium according to a Griess reaction (20). The accumulated interleukin-6 (IL-6) and prostaglandin E2 (PGE2) in the culture medium were measured using commercial ELISA kits (R\&D, USA) according to the manufacturer's instructions.

To evaluate the protective effect of candidate BECCs on cardiomyocytes against simulated ischemia injury, a rat $\mathrm{H} 9 \mathrm{c} 2$ cardiomyoblasts simulated ischemia model was established (17). After overnight incubation, the cells were pretreated for $24 \mathrm{~h}$ with various samples (corresponding concentrations in $0.1 \mathrm{mg} / \mathrm{ml}$ Cardiotonic Pill). To mimic the in viwo conditions of myocardial ischemia, the cells were incubated in a buffer containing $116.4 \mathrm{mM} \mathrm{NaCl}, 5.4 \mathrm{mM} \mathrm{KCl}, 1.8 \mathrm{mM} \mathrm{CaCl}_{2}$, $0.8 \mathrm{mM} \mathrm{MgSO}_{4}, 2.6 \mathrm{mM} \mathrm{NaH}{ }_{2} \mathrm{PO}_{4}, 26.2 \mathrm{mM} \mathrm{NaHCO}$, 20.1 $\mathrm{mM}$ HEPES and then exposed to $2 \% \mathrm{O}_{2}-93 \% \mathrm{~N}_{2}-5 \%$ $\mathrm{CO}_{2}$ for $6 \mathrm{~h}$. After the treatment, cell viability was determined by CCK-8 assay (Dojindo Molecular Technologies Inc., Japan), according to the manufacturer's instructions. Cells cultured in complete medium under a normoxic atmosphere served as a control. The treatment concentrations were chosen based on the optimal concentration of Cardiotonic Pill (Figure S2, Supplementary Material).

\section{Bioactivity Assays In Vivo}

To further investigate whether the candidate BECCs (III) are a bioactive equivalent with original formulation in terms of in vivo efficacy, we utilized a rat model of MI (21-24). All animal procedures were approved by the Animal Ethics Committee of China Pharmaceutical University. Male SpragueDawley rats (250-300 g) were purchased from the Aier Maite Technology Corporation (Suzhou, China). The rats were housed in humidity- and temperature- controlled environment with a 12 h light: 12 h dark cycle. Water and standard laboratory diet were available ad libitum.

MI was produced by the ligation of the left anterior descending (LAD) coronary artery as reported previously $(21,22)$. Rats were randomly assigned into seven groups: four treatment groups (CP, candidate BECGs (III), mixture of reference compounds (MRCs) (III), remaining part (III)), a MI group, and a sham group. Briefly, various samples (corresponding concentrations in $20 \mathrm{mg} / \mathrm{kg}$ Cardiotonic Pill) in the treatment group were given once daily by oral administration for 3 days before MI. The sham and MI group received only saline. For MI operation, rats were anesthetized with chloral hydrate $(300 \mathrm{mg} / \mathrm{kg}$ i.p.) and ventilated by a respirator $(\mathrm{HX}$ 100E, Taimeng Co. Ltd., China) with a tidal volume of $10 \mathrm{ml} / \mathrm{kg}$ and a respiratory rate of 80 cycles per minute. A left thoracotomy was performed in the fourth intercostal space, and then MI was induced by ligation of the LAD artery $2 \mathrm{~mm}$ from the tip of the left auricle. The sham-operated rats underwent the same thoracotomy without LAD ligation. Electrocardiogram was recorded before and after operation procedures. Coronary occlusion was confirmed by ST segment 
elevation in the electrocardiogram and the appearance of epicardial cyanosis.

To evaluate the bioactivity of candidate BECGs (III) on cardiac function, left ventricular (LV) catheterization was performed (25). At the end of $6 \mathrm{~h}$ ischemic period, a catheter was inserted into the left ventricle for evaluating cardiac function. Left ventricular end diastolic pressure (LVEDP), left ventricular systolic pressure (LVSP), $+d P / d t$ and $-d P / d t$ were recorded through a biological mechanic experiment system (BL420, Taimeng Co. Ltd., China). Cardiac marker enzymes including creatine kinase-MB (CK-MB) and $\mathrm{LDH}$ were tested for estimation of myocardial cell damage using commercial kits (Jiancheng Bioengineering Institute, China) (26). To investigate the bioactivity of candidate BECGs (III) on inflammatory response, tumor necrosis factor- $\alpha$ (TNF- $\alpha)$ level in serum was measured using a commercial ELISA kit (R\&D, USA) (27). At the end of experiment, the heart was excised, sliced into five sections, and infarct size was measured using 2, 3, 5-triphenyltetrazolium chloride (TTG) staining to assess myocardial injury as reported previously $(28,29)$. Sections were photographed and the area at risk was quantified with the aid of the Image-Pro Plus 6 software.

\section{Metabolomic Profiling}

To validate the assessment results of bioactive equivalence between BECCs and original CP, metabolomics strategy was applied to profile the metabolites in rat myocardial tissues using gas chromatography/time-of-flight mass spectrometry (GC/TOF MS) coupled to partial least square-discriminant analysis (PLS-DA). Rat hearts $(n=4-6)$ were immediately snap-frozen in liquid nitrogen and stored at $-80^{\circ} \mathrm{C}$ until metabolomic profiling. The same extraction, derivatization, and analysis procedures were applied as described previously (30,31). Myocardial tissue (20 mg) from infart area was homogenized in a tube containing $800 \mu \mathrm{l}$ methanol. [1, 2- ${ }^{13} \mathrm{C}_{2}$ ] Myristic acid was added to each tube as the internal standard. With an Agilent 7683 autosampler, the derivatized samples were analyzed using an Agilent 6890N GC system (Agilent, USA) coupled with a Pegasus III mass spectrometer (Leco, USA). The injector temperature was set at $270^{\circ} \mathrm{C}$ and helium was used as carrier gas at a constant flow rate of $1 \mathrm{ml} / \mathrm{min}$. Column temperature was initially maintained at $70^{\circ} \mathrm{C}$ for $2 \mathrm{~min}$ and then was increased at a rate of $35^{\circ} \mathrm{C} / \mathrm{min}$ to $305^{\circ} \mathrm{C}$, where it was held for $2 \mathrm{~min}$. The transfer line temperature was set at $250^{\circ} \mathrm{C}$ and ion source temperature at $200^{\circ} \mathrm{C}$ for the mass spectrometer. A 70-eV electron beam at a current of $2.0 \mathrm{~mA}$ were used for ions generation. The GC/TOF MS data of metabolites profiling of myocardial tissue were analyzed by PLS-DA, using the SIMCA-P 11.0 (Umetrics, Sweden). For PLS-DA modeling, samples were divided into different groups (e.g., sham, MI, BECGs and CP) as the qualitative "dummy" variable, $\mathrm{Y}$.

\section{Statistical Analysis}

All bioactive data were presented as mean \pm standard deviation (SD). Data were subjected to statistical analysis using Graphpad Prism 6.0. One-way analysis of variance (ANOVA) with Dunnett's post-hoc test was carried out for statistical comparison. In all cases, a $P$-value $<0.05$ was considered to be significant.

For bioactive equivalence assessment, all the bioassay results were transformed to efficacy value according to the following equation:

$$
\text { Efficacy }_{\text {drug }}=\frac{\operatorname{Abs}\left(\text { Activity }_{\text {drug }}-\text { Activity }_{\text {model }}\right)}{\operatorname{Abs}\left(\text { Activity }_{\text {control }}-\text { Activity }_{\text {model }}\right)} \times 100 \%
$$

where Activitydrug, Activity model $_{\text {and }}$ Activity control $_{\text {were the measured }}$ values of drug group (candidate BECGs, MRGs or original CP treated), model group (Ox-LDL-mediated HUVEC injury, LPS-stimulated RAW 264.7 macrophage inflammation, simulated ischemia-induced $\mathrm{H} 9 \mathrm{c} 2$ cardiomyocytes injury or myocardial infarction) and control group respectively in a given bioassay. Bioactive equivalence was evaluated by calculating 90\% confidence interval (CI) of the ratio between the efficacies of candidate BECGs and CP (two one-sided $t$ test, see more information in Supplementary Material). If the $90 \%$ CI of relative efficacy compared to original CP fell within the range of $70-143 \%$ (18), the candidate BECGs were considered to be a bioactive equivalent with original CP.

\section{RESULTS}

\section{Chemical Profiling of CP}

A variety of components were detected at HPLC-UV chromatogram using the segmental monitoring method (Fig. 1a). Structural characterization was performed by Q-TOF MS, and the total ion chromatograms of CP in positive and negative ion modes were shown in Figure S3, Supplementary Material. In total, 36 compounds in CP were detected and identified by comparison with available reference compounds or literature information, including 13 phenolic acids, 15 saponins and 8 tanshinones (Table I) $(32,33)$.

\section{Determination of Candidate BECCs from CP}

In our experiment, normalized peak area ratio $(\%)$ was chosen as the selection criterion. According to the HPLC chromatogram of CP, the selection threshold was set at $10 \%$ of the total peak area in the first round and only one peak, identified as tanshinol, was trapped and prepared, labeled as candidate BECCs (I) (Fig. 1b). 
When the selection threshold was reduced to $1 \%$ or $0.1 \%, 10$ or 18 peaks were prepared as candidate BECCs (II) or candidate BECGs (III), respectively (Fig. 1c and d). Candidate BECGs (II) mainly consisted of phenolic acids, while candidate BECGs (III) consisted of 10 phenolic acids, 4 saponins and 4 tanshinones. Remaining part (III) of CP without candidate BECGs (III) mainly consisted of highly polar compounds such as carbohydrates, and other minor constituents.

\section{Trapping and Preparing Candidate BECCs}

Three batches of candidate BECGs based on the described selection threshold were prepared through the corresponding collection programs. Using a semi-preparative column, the system could provide samples at the milligram level per HPLC run, which was adequate for cell tests. For our experiments in rats, a 1 week preparation period was required to accumulate

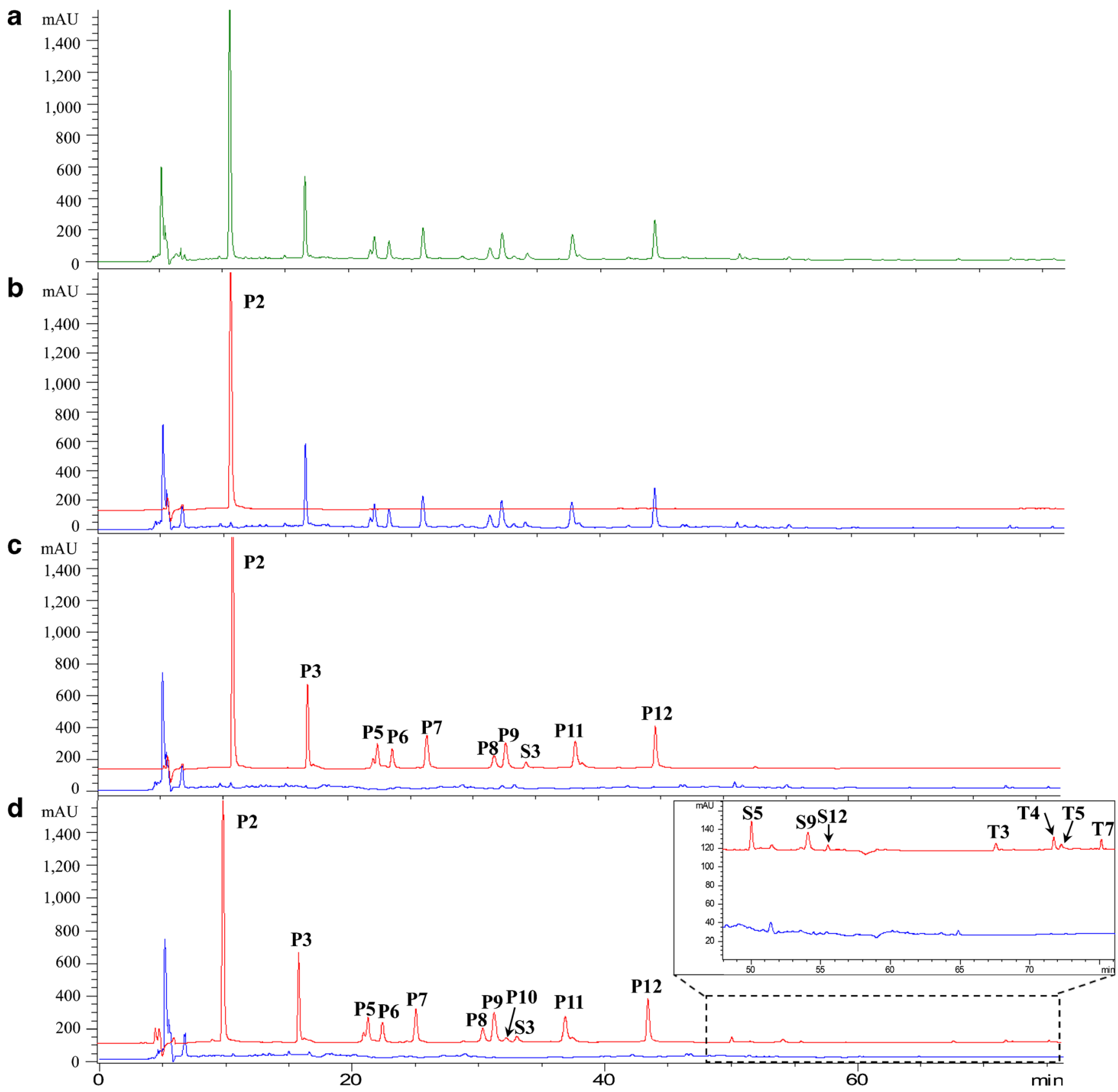

Fig. I Validation of candidate BECCs in the three rounds of screening by HPLC-UV. Samples of Cardiotonic Pill (depicted in green), candidate BECCs (red) and the remaining part (blue) were analyzed at the same concentration. UV wavelengths: $203 \mathrm{~nm}$ for 0-57 min; 28I nm for 57-77 min. The peaks in the chromatograms are assigned the same numbers as in Figure S3, Supplementary Material. (a) Chromatogram of Cardiotonic Pill. (b) Chromatogram of candidate BECCs (I) and remaining part (I). Candidate BECCS (I) consisted of one compound, identified as tanshinol. (c) Chromatogram of candidate BECCs (II) and remaining part (II). Candidate BECCs (II) consisted of IO compounds (mainly phenolic acids). (d) Chromatogram of candidate BECCs (III) and remaining part (III). Candidate BECCs (III) consisted of I 8 compounds, including 10 phenolic acids, 4 saponins and 4 tanshinones. BECCs bioactive equivalent combinatorial components. 
Table I The Retention Time, MS Data and Characterization of Identified Phenolic Acids, Saponins and Tanshinones in Cardiotonic Pill

\begin{tabular}{|c|c|c|c|c|c|c|}
\hline Peak no. & $t_{R}(\min )$ & {$[\mathrm{M}-\mathrm{H}]^{-}(\mathrm{m} / \mathrm{z})$} & Theoretical $(\mathrm{m} / \mathrm{z})$ & Error (ppm) & Formula & Identification \\
\hline $\mathrm{Pl}$ & 8.85 & 197.0450 & 197.0455 & -2.77 & $\mathrm{C}_{9} \mathrm{H}_{10} \mathrm{O}_{5}$ & (s)-3-(3,4-dihydroxyphenyl) lactic acid \\
\hline$P 2^{\mathrm{a}}$ & 9.76 & 197.0453 & 197.0455 & -1.25 & $\mathrm{C}_{9} \mathrm{H}_{10} \mathrm{O}_{5}$ & Tanshinol \\
\hline$P 3^{\mathrm{a}}$ & 15.38 & 137.0246 & | 37.0244 & 1.33 & $\mathrm{C}_{7} \mathrm{H}_{6} \mathrm{O}_{3}$ & Protocatechuic aldehyde \\
\hline$P 4^{\mathrm{a}}$ & 16.60 & | 79.035 | & 179.0350 & 0.66 & $\mathrm{C}_{9} \mathrm{H}_{8} \mathrm{O}_{4}$ & Caffeic acid \\
\hline$P 5^{\mathrm{a}}$ & 20.70 & 537.1032 & 537.1038 & 1.12 & $\mathrm{C}_{27} \mathrm{H}_{22} \mathrm{O}_{12}$ & Isolithospermic acid A \\
\hline$P 6^{a}$ & 21.89 & 537.1041 & 537.1038 & -0.34 & $\mathrm{C}_{27} \mathrm{H}_{22} \mathrm{O}_{12}$ & Isolithospermic acid B \\
\hline$P 7^{\mathrm{a}}$ & 24.73 & 417.0830 & 417.0827 & 0.67 & $\mathrm{C}_{20} \mathrm{H}_{18} \mathrm{O}_{10}$ & Salvianolic acid D \\
\hline$P 8^{a}$ & 29.40 & 339.0518 & 339.0510 & 2.28 & $\mathrm{C}_{18} \mathrm{H}_{12} \mathrm{O}_{7}$ & Salvianolic acid G \\
\hline $\mathrm{Pg}^{\mathrm{a}}$ & 30.30 & 359.0780 & 359.0772 & 2.11 & $\mathrm{C}_{18} \mathrm{H}_{16} \mathrm{O}_{8}$ & Rosmarinic acid \\
\hline $\mathrm{P} 10^{\mathrm{a}}$ & 30.67 & 537.1045 & 537.1038 & 1.58 & $\mathrm{C}_{27} \mathrm{H}_{22} \mathrm{O}_{12}$ & Lithospermic acid \\
\hline$S I^{\mathrm{a}}$ & 31.04 & 977.5309 & 977.5327 & 1.62 & $\mathrm{C}_{47} \mathrm{H}_{80} \mathrm{O}_{18}$ & Notoginsenoside-RI \\
\hline$S 2^{\mathrm{a}}$ & 33.82 & 991.5497 & 991.5483 & -1.47 & $\mathrm{C}_{48} \mathrm{H}_{82} \mathrm{O}_{18}$ & Ginsenoside-Re \\
\hline$S 3^{\mathrm{a}}$ & 34.15 & 845.4912 & 845.4904 & -1.09 & $\mathrm{C}_{42} \mathrm{H}_{72} \mathrm{O}_{14}$ & Ginsenoside-RgI \\
\hline $\mathrm{P} \mid I^{\mathrm{a}}$ & 34.91 & $7|7.146|$ & 717.1465 & -0.55 & $\mathrm{C}_{36} \mathrm{H}_{30} \mathrm{O}_{16}$ & Salvianolic acid B \\
\hline $\mathrm{P} I 2^{\mathrm{a}}$ & 41.63 & 493.1146 & 493.1140 & -1.21 & $\mathrm{C}_{26} \mathrm{H}_{22} \mathrm{O}_{10}$ & Salvianolic acid A \\
\hline $\mathrm{P} \mid 3^{\mathrm{a}}$ & 46.67 & 491.0990 & 491.0984 & 1.28 & $\mathrm{C}_{26} \mathrm{H}_{20} \mathrm{O}_{10}$ & Salvianolic acid C \\
\hline$S 4^{\mathrm{a}}$ & 50.10 & 845.4899 & 845.4904 & -0.01 & $\mathrm{C}_{42} \mathrm{H}_{72} \mathrm{O}_{14}$ & Ginsenoside-Rf \\
\hline$S 5^{\mathrm{a}}$ & 52.13 & I, I53.6005 & I,I53.60| | & 1.04 & $\mathrm{C}_{54} \mathrm{H}_{92} \mathrm{O}_{23}$ & Ginsenoside-Rb | \\
\hline $56^{a}$ & 52.76 & 815.4792 & 815.4798 & 1.12 & $\mathrm{C}_{41} \mathrm{H}_{70} \mathrm{O}_{13}$ & Notoginsenoside-R2 \\
\hline $\mathrm{S} 7^{\mathrm{a}}$ & 53.63 & 829.4951 & 829.4955 & 0.48 & $\mathrm{C}_{42} \mathrm{H}_{72} \mathrm{O}_{13}$ & Ginsenoside-Rg2 \\
\hline S8 & 54.43 & I, I23.5878 & I, I23.5906 & 2.61 & $\mathrm{C}_{53} \mathrm{H}_{90} \mathrm{O}_{22}$ & Ginsenoside-Rb3 \\
\hline $59^{a}$ & 55.15 & 683.4379 & 683.4376 & -0.56 & $\mathrm{C}_{36} \mathrm{H}_{62} \mathrm{O}_{9}$ & Ginsenoside-Rh I \\
\hline $\mathrm{S} 10^{\mathrm{a}}$ & 55.56 & I, I 23.5907 & I, I23.5906 & -1.31 & $\mathrm{C}_{53} \mathrm{H}_{90} \mathrm{O}_{22}$ & Ginsenoside-Rb2 \\
\hline $\mathrm{S} I 1^{\mathrm{a}}$ & 56.07 & 683.4370 & 683.4376 & 0.98 & $\mathrm{C}_{36} \mathrm{H}_{62} \mathrm{O}_{9}$ & Ginsenoside-FI \\
\hline $\mathrm{S} 12^{\mathrm{a}}$ & 57.21 & 991.5507 & 991.5483 & -2.39 & $\mathrm{C}_{48} \mathrm{H}_{82} \mathrm{O}_{18}$ & Ginsenoside-Rd \\
\hline $\mathrm{S} 13^{\mathrm{a}}$ & 59.76 & 829.4959 & 829.4955 & -0.20 & $\mathrm{C}_{42} \mathrm{H}_{72} \mathrm{O}_{13}$ & Ginsenoside-F2 \\
\hline $\mathrm{S} 14^{\mathrm{a}}$ & 60.45 & 829.4963 & 829.4955 & -0.88 & $\mathrm{C}_{42} \mathrm{H}_{72} \mathrm{O}_{13}$ & 20(S)-Ginsenoside Rg3 \\
\hline $\mathrm{S} 15^{\mathrm{a}}$ & 60.78 & 829.4936 & 829.4955 & 2.18 & $\mathrm{C}_{42} \mathrm{H}_{72} \mathrm{O}_{13}$ & 20(R)-Ginsenoside Rg3 \\
\hline $\mathrm{TI}^{\mathrm{a}}$ & 62.10 & 311.1288 & 311.1278 & 2.84 & $\mathrm{C}_{19} \mathrm{H}_{18} \mathrm{O}_{4}$ & Tanshinone IIB \\
\hline $\mathrm{T} 2$ & 63.61 & 337.1429 & 337.1434 & 1.70 & $\mathrm{C}_{21} \mathrm{H}_{20} \mathrm{O}_{4}$ & Danshenxinkun D \\
\hline$T 3^{\mathrm{a}}$ & 66.81 & 279.1021 & 279.1016 & 1.86 & $\mathrm{C}_{18} \mathrm{H}_{14} \mathrm{O}_{3}$ & | 5, | 6-Dihydrotanshinone | \\
\hline $\mathrm{T} 4^{\mathrm{a}}$ & 71.01 & 277.0864 & 277.0859 & 2.96 & $\mathrm{C}_{18} \mathrm{H}_{12} \mathrm{O}_{3}$ & Tanshinone I \\
\hline $\mathrm{T} 5^{\mathrm{a}}$ & 71.55 & 297.1496 & 297.1485 & 2.75 & $\mathrm{C}_{19} \mathrm{H}_{20} \mathrm{O}_{3}$ & Cryptotanshinone \\
\hline$T 6^{\mathrm{a}}$ & 72.92 & 279.1023 & 279.1016 & -2.56 & $\mathrm{C}_{18} \mathrm{H}_{14} \mathrm{O}_{3}$ & Methylenetanshinquinone \\
\hline$T 7^{\mathrm{a}}$ & 74.48 & 295.1334 & 295.1329 & 1.77 & $\mathrm{C}_{19} \mathrm{H}_{18} \mathrm{O}_{3}$ & Tanshinone IIA \\
\hline$T 8^{\mathrm{a}}$ & 75.01 & 283.1704 & 283.1693 & -4.07 & $\mathrm{C}_{19} \mathrm{H}_{22} \mathrm{O}_{2}$ & Miltirone \\
\hline
\end{tabular}

PI_PI 3 phenolic acids; SI-SI 5 saponins; TI-T8 tanshinones. tR: retention time

${ }^{a}$ Compared with reference compounds

sufficient samples using this system. The prepared samples were dried and reconstituted with $75 \%(\mathrm{v} / \mathrm{v})$ ethanol to the original concentration for HPLC validation. As shown in Fig. 1, candidate BECGs were trapped and prepared from CP completely.

The preparation process may potentially lead to sample loss because of possible adsorption to the chromatographic column and compound degradation during heating processes. Thus, we investigated the preparative recovery and repeatability for each compound in the candidate BECCs (Table S1, Supplementary Material). The preparative recovery ranged from $87.7 \%$ to $97.9 \%$, while the average recovery rate was approximately $92.9 \%$. The preparative process showed stable repeatability with a $\mathrm{RSD}<6.6 \%$. This result indicates that the described system is feasible and reliable.

\section{In Vitro Assessment of Bioactive Equivalence Between Candidate BECCs and Original CP}

CP showed remarkable protective effects in the three cell models, while candidate BECGs (I) exerted weak effects 


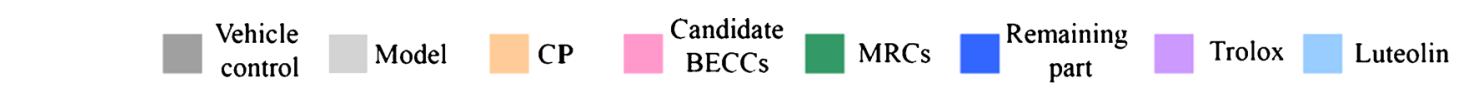

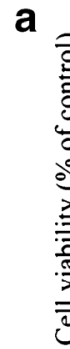
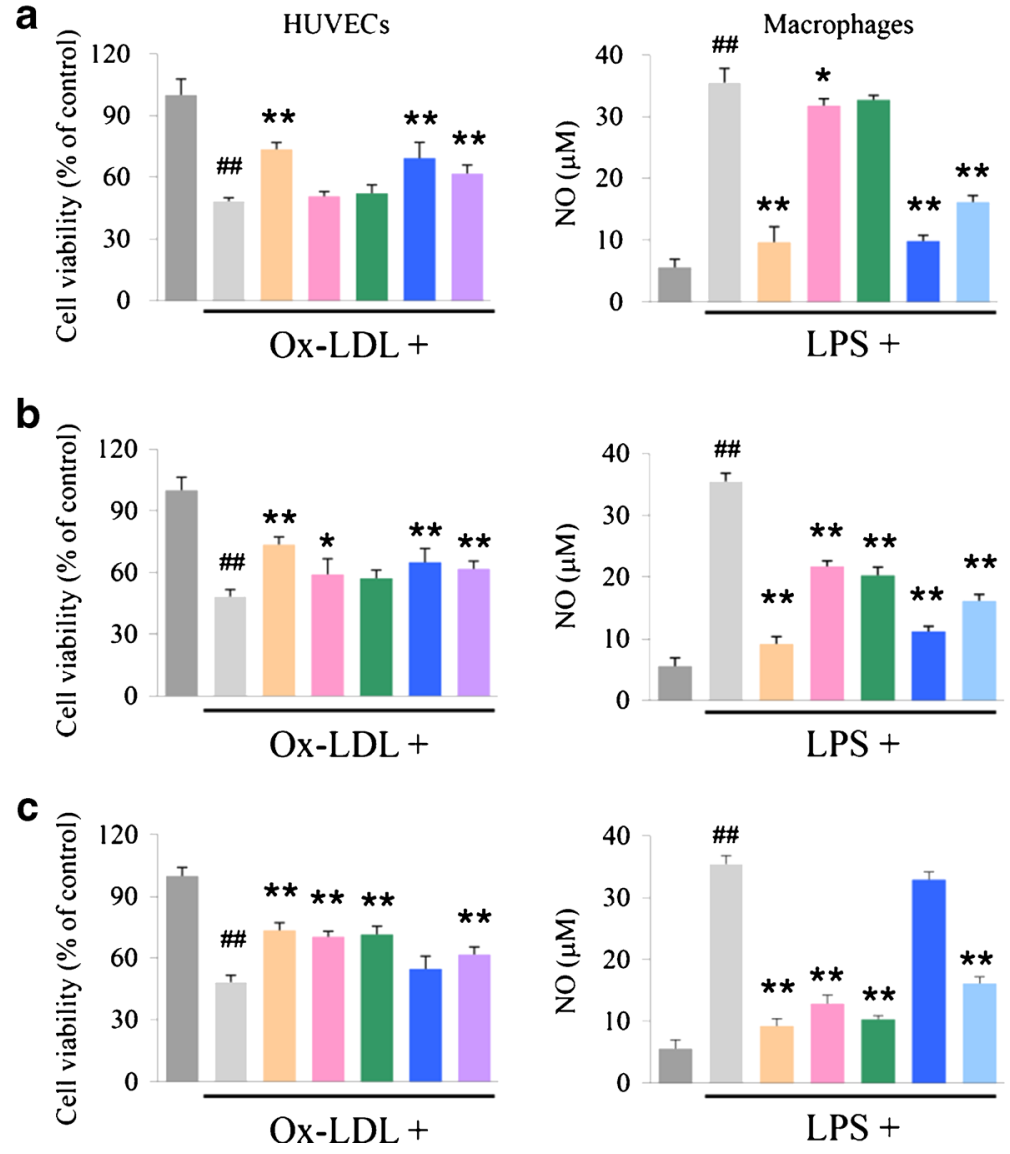

Fig. 2 In vitro activity assays of candidate BECCs in the three rounds of screening. Samples in (a) the first round of screening, (b) the second round of screening, (c) the third round of screening were investigated in three cell models. HUVEC injury was induced by incubation with Ox-LDL and cell viability (as \% of control) was assayed by CCK-8. Effects of samples on NO production of LPS-activated murine macrophage RAW264.7 cells was determined by Griess reaction. After 6 h of simulated ischemia, cell viability of $\mathrm{H} 9 \mathrm{c} 2$ cardiomyoblasts was assayed by CCK-8. Trolox and luteolin were used as a positive control in HUVECs and macrophages respectively. Results are expressed as mean $\pm \mathrm{SD}$ of at least three independent experiments. $* P<0.05$, *** $P<0.0 \mathrm{I}$ versus model group. \#\# $P<0.0$ I versus vehicle control group (one-way ANOVA, Dunnett test). Cells in model group were untreated. CP Cardiotonic Pill; BECCs bioactive equivalent combinatorial components; MRCs mixture of reference compounds; Remaining part: CP lacking candidate BECCs; Ox-LDL oxidized low density lipoprotein; LPS lipopolysaccharide; SI simulated ischemia.

(Fig. 2a) and could not achieve bioactive equivalence as compared to the original CP (Table II). As such, we adjusted our feedback loop by reducing the selection threshold to $1 \%$ of total peak area and performed the second round of screening. Candidate BECGs (II) comprised of 10 compounds showed some activities (Fig. 2b). However, in the bioactive equivalence assessment, candidate BECCs (II) failed to meet the 70143\% requirements (Table II). For example, we observed a notable anti-inflammatory effect of candidate BECGs (II) in macrophages compared with model group (Fig. 2b, $P<0.0001, n=3$, one-way ANOVA, Dunnett test), but $90 \%$ CI of efficacy in suppressing the production of nitric oxide (NO) was $49.0-56.0 \%$, and demonstrated that candidate BECGs (II) could not achieve bioactive equivalence with CP. In the third round of screening, the selection threshold was further reduced to $0.1 \%$ of total peak area, candidate BECGs (III) of 18 compounds increased the cell viability of damaged HUVECs and cardiomyocytes, and also alleviated the LPS-induced increase of $\mathrm{NO}$ in macrophages compared with model group (Fig. 2c, in HUVECs, $P<0.0001$; in macrophages, $P<0.0001$; in cardiomyocytes, $P<0.0001, n=3$, one-way ANOVA, Dunnett test). In the bioactive equivalence assessment, 90\% CI for the efficacies of candidate BECGs (III) in all the cell tests fell within 70-143\%, as listed in Table II. At the same time, the low activity of remaining part (III) also suggested that most of the bioactive components of CP were included in candidate BECCs (III).

Ox-LDL increased LDH release and reactive oxygen species (ROS) concentration in HUVECs substantially, whereas preincubation with CP or candidate BECGs (III) notably 
Table II Bioactive Equivalence Evaluation of Candidate BECCs and MRCs by In Vitro Assays

\begin{tabular}{|c|c|c|c|c|c|c|}
\hline In vitro assay & Candidate BECCs (I) & MRCs (I) & Candidate BECCs (II) & MRCs (II) & Candidate BECCs (III) & MRCs (III) \\
\hline \multicolumn{7}{|l|}{ HUVECS } \\
\hline Cell viability & $2.1-13.2 \%$ & 1.5-23.6\% & $20.7-58.8 \%$ & $23.7-44.8 \%$ & $77.0-100.4 \%$ & $77.8-107.7 \%$ \\
\hline $\mathrm{LDH}$ & - & - & - & - & $79.6-93.6 \%$ & $73.5-91.1 \%$ \\
\hline ROS & - & - & - & - & $78.6-85.1 \%$ & $73.7-79.0 \%$ \\
\hline \multicolumn{7}{|l|}{ Macrophages } \\
\hline NO & $10.5-18.0 \%$ & $8.4-13.0 \%$ & $49.0-56.0 \%$ & $53.1-63.1 \%$ & $80.6-92.3 \%$ & $91.8-100.4 \%$ \\
\hline IL-6 & - & - & - & - & $81.7-84.1 \%$ & $78.6-81.0 \%$ \\
\hline PGE2 & - & - & - & - & $\mid 20.1-150.0 \%$ & $|47|-.\mid 85.3 \%$ \\
\hline \multicolumn{7}{|c|}{ Cardiomyocytes } \\
\hline Cell viability & $38.3-47.8 \%$ & 13.7-52.1\% & $22.5-27.3 \%$ & $8.2-23.0 \%$ & $75.6-105.9 \%$ & $77.3-104.8 \%$ \\
\hline
\end{tabular}

$n=3$. Bioactive equivalence was evaluated by two one-sided $t$ test. The $90 \% \mathrm{Cl}$ of relative efficacy of candidate BECCs and MRCs compared to original CP was showd in the table. If the $90 \% \mathrm{Cl}$ of relative efficacy fell within the range of $70-143 \%$, the candidate BECCs were considered to be a bioactive equivalent with original Cardiotonic Pill

BECCs bioactive equivalent combinatorial components; MRCs mixture of reference compounds

attenuated the Ox-LDL-induced damage (Fig. 3a, CP versus Ox-LDL group, $P<0.0001$ for $\mathrm{LDH}$ assay, $P<0.0001$ for ROS assay; candidate BECCs (III) versus Ox-LDL group, $P<0.0001$ for LDH assay, $P<0.0001$ for ROS assay, $n=3$, one-way ANOVA, Dunnett test). Additionally, results were consistent with the cell viability assay (Fig. 2c). To investigate the biological activities on macrophages, LPS stimulation resulted in a substantial increase of IL-6 and PGE2 secretion, while preincubation of CP or candidate BECGs (III) alleviated the LPS-induced increase of these inflammatory factors (Fig. 3b, CP versus LPS group, $P<0.0001$ for IL-6 assay, $P<0.0001$ for
PGE2 assay; candidate BECGs (III) versus LPS group, $P<0.0001$ for IL-6 assay, $P<0.0001$ for PGE2 assay, $n=3$, one-way ANOVA, Dunnett test). The results suggested that candidate BECGs (III) had a similar efficacy to CP.

In the bioactive equivalence assessment, 90\% CI showed that ratios of efficacies between $\mathrm{CP}$ and candidate BECCs (III) in most cases lay within the acceptance range of 70-143\% (Table II). Thus, candidate BECGs (III) could be considered as BECGs of CP in vitro.

To exclude the contribution of any undetected peaks masked in the candidate BECGs collected, we prepared a
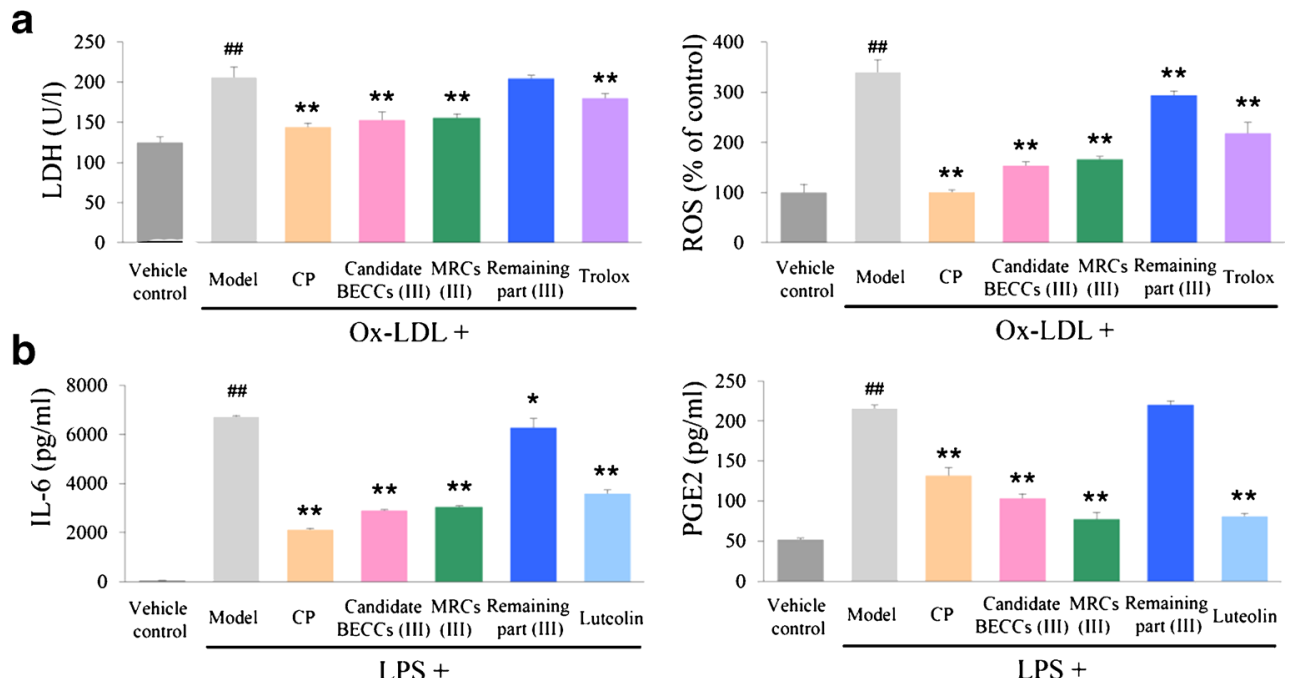

Fig. 3 Effects of Candidate BECCS (III) on LDH and ROS in HUVECs and IL-6 and PGE2 in macrophages. (a) HUVECs were incubated with vehicle, CP, candidate BECCs (III), MRCs (III), remaining part (III) and trolox for 12 h, following 24-h stimulation of Ox-LDL. Supematant of HUVECs was collected for LDH assay by a commercial kit. ROS production (as \% of increase) in HUVECs was determined by a fluorometric assay using DCFH-DA as a probe. (b) Macrophages were pretreated with vehicle, CP, candidate BECCs (III), MRCs (III), remaining part (III) and luteolin for $2 \mathrm{~h}$, following 20-h stimulation of LPS. The accumulated IL-6 and PGE2 in the culture medium were measured using commercial ELISA kits. Trolox and luteolin were used as a positive control in HUVECs and macrophages respectively. Results are expressed as mean $\pm \mathrm{SD}$ of at least three independent experiments. ${ }^{*} P<0.05$, $* * P<0.0$ I versus model group. \#\# $P<0.0$ I versus vehicle control group (one-way ANOVA, Dunnett test). Cells in model group were untreated. CP Cardiotonic Pill; BECCs bioactive equivalent combinatorial components; MRCs mixture of reference compounds; Remaining part: CP lacking candidate BECCs; Ox-LDL oxidized low density lipoprotein; LPS lipopolysaccharide. 
mixture of reference compounds (MRCs) with the identical compositions of candidate BECGs (Table S2, Supplementary Material) for bioactivity validation. Similar with candidate BECGs (III), MRCs (III) showed marked protective effects in all three cell models (Figs. 2c and 3). The bioactive equivalence assessment data were also consistent with that of candidate BECCs (III); 90\% CI of efficacies in most cases for MRCs (III) lay within 70-143\% (Table II).

When the 18 compounds in candidate BECGs (III) were given individually at the concentration as in the original CP, none of these compounds showed substantial protective effects on HUVEGs (Fig. 4a). For example, tanshinol, one of the most abundant compounds in CP, demonstrated weak protective effects on HUVECs at the same dose level as in the formulation $(10.7 \mu \mathrm{g} / \mathrm{ml})$. When the dose increased to $40 \mu \mathrm{g} / \mathrm{ml}$, tanshinol showed some activity on HUVEGs (Fig. 4b, tanshinol versus Ox-LDL group, $P=0.0027, n=3$, one-way ANOVA, Dunnett test), but it was not able to achieve bioactive equivalence with $\mathrm{CP}$, even up to $100 \mu \mathrm{g} / \mathrm{ml}$. Candidate BECCs (III) consisted of three groups of ingredients, including 10 phenolic acids, 4 saponins and 4 tanshinones. In order to test whether each group of constituents is necessary, phenolic acids, saponins or tanshinones were removed from candidate BECCs (III) respectively, and the combination of any two groups could not show equivalent bioactivity to original CP (Figure S4, Supplementary Material). The results indicated that each group of ingredients was indispensable.

\section{In Vivo Assessment of Bioactive Equivalence Between Candidate BECCs and Original CP}

TTC-stained hearts were shown in Fig. 5a. Pretreatment with CP significantly reduced the infarct size compared with the MI group (Fig. 5b, 29.4 $\pm 4.0 \%$ versus $53.3 \pm 3.7 \%, P=$ $0.0016, n=5$, one-way ANOVA, Dunnett test). Candidate BECGs (III) showed a similar effect of decreasing the infarct size to $29.4 \pm 3.0 \%$ (Fig. 5b, $P=0.0016, n=5$, one-way ANOVA, Dunnett test). MI resulted in marked elevation in the serum levels of CK-MB and LDH (Fig. 5c and d). CP and candidate BEGCs (III) inhibited the MI-induced increases in the activities of these enzymes (Fig. 5b, CP versus MI group, $P=0.0004$ for CK-MB assay, $P=0.0017$ for $\mathrm{LDH}$ assay; candidate BECCs (III) versus MI group, $P<0.0001$ for $\mathrm{CK}-\mathrm{MB}$
Fig. 4 Protective effects of compounds in BECCs on HUVECs against Ox-LDL injury. HUVEC injury was induced by pretreatment with Ox-LDL for $24 \mathrm{~h}$ and cell viability (as \% of control) was assayed by CCK-8. (a) Protective effects of 18 individual compounds. The concentrations of 18 compounds were equal to their concentrations in $0.4 \mathrm{mg} / \mathrm{ml}$ Cardiotonic Pill. Compound P2 is tanshinol with a concentration of $10.7 \mu \mathrm{g} / \mathrm{ml}$ (marked in red). (b) Protective effects of tanshinol on HUVECs at different doses. Results are expressed as mean $\pm \mathrm{SD}$ of at least three independent experiments. ${ }^{*} * P<0.0$ I versus Ox-LDL group. \#\# $P<0.01$ versus vehicle control group (oneway ANOVA, Dunnett test). Cells in model group were untreated. $B E C C$ s bioactive equivalent combinatorial components; $C P$ Cardiotonic Pill; Ox-LDL oxidized low density lipoprotein. a

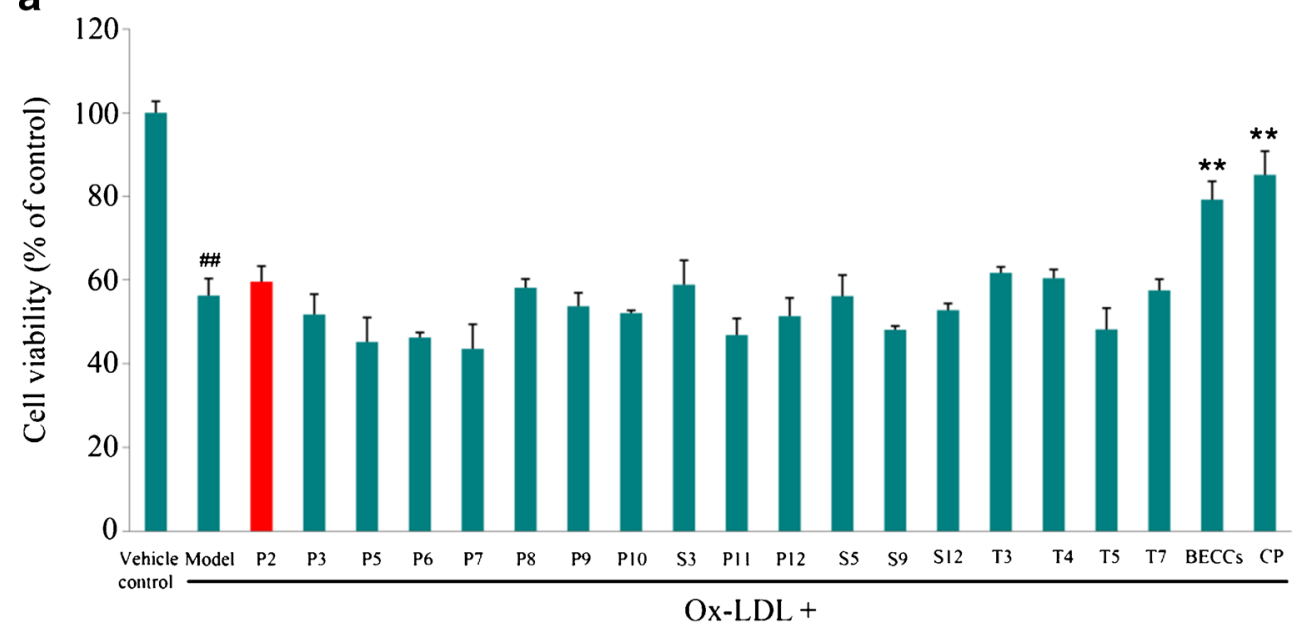

b

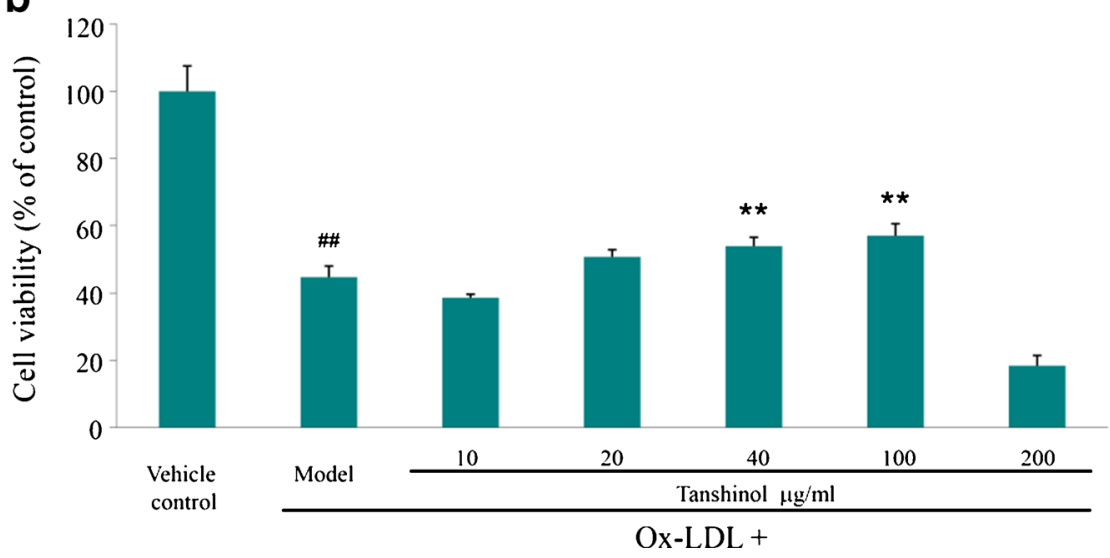


a

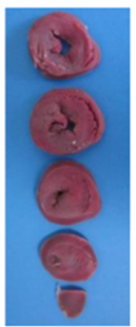

Sham

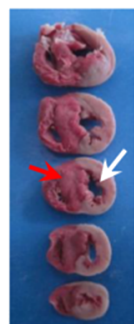

MI

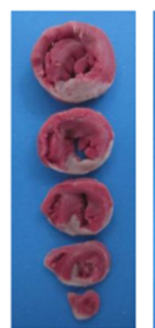

CP

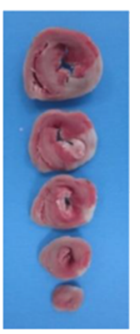

Candidate

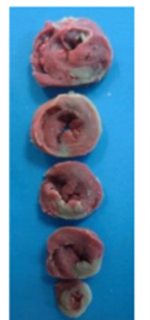

MRCs

MRCs
(III)

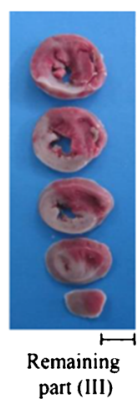

b

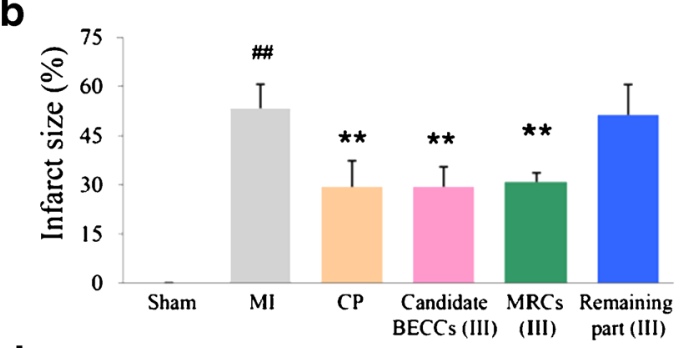

d

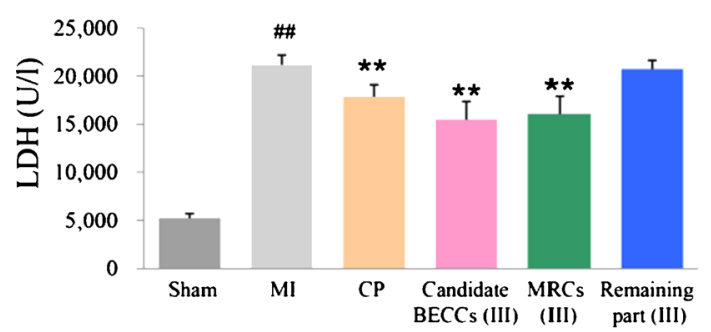

f

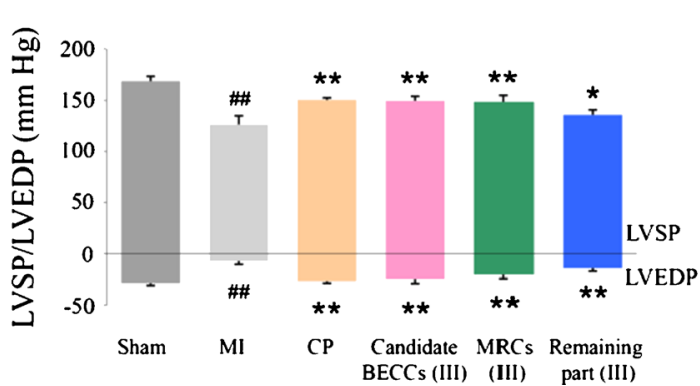

C

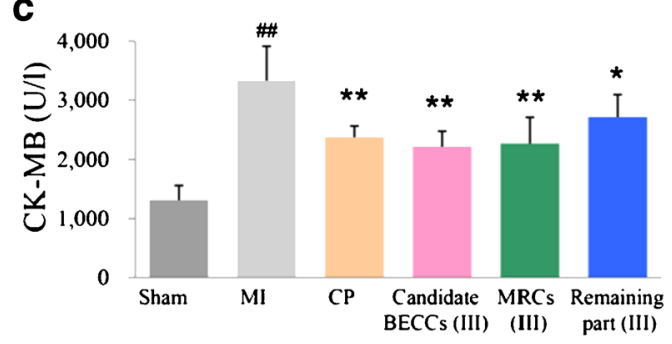

e

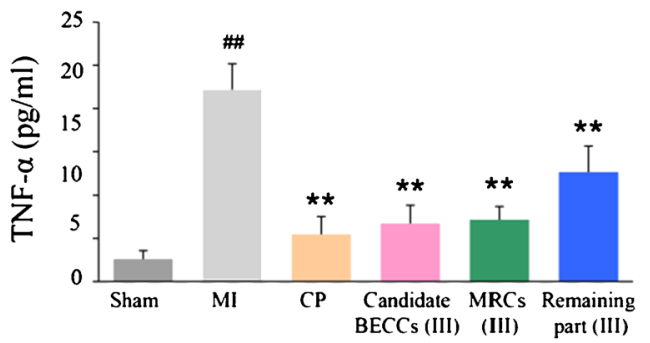

g

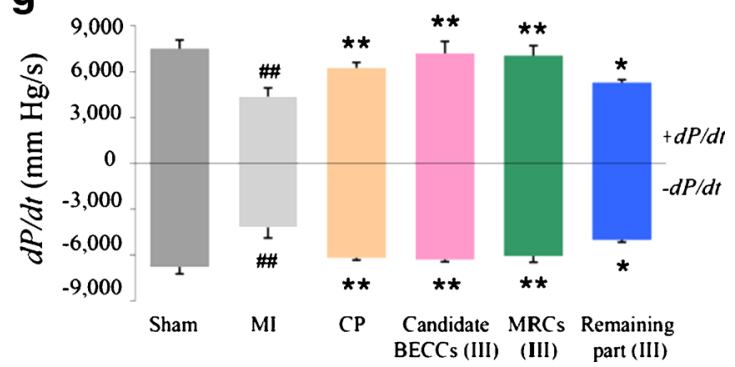

Fig. 5 Candidate BECCs (III) showed comparable cardio-protective effects with CP against myocardial infarction. (a) Representative pictures of 2, 3, 5triphenyltetrazolium chloride-stained myocardial sections (scale bar, $0.5 \mathrm{~cm}$ ). Red arrow indicates non-infarct region while white arrow indicates infarct region. $n=5$. (b) Quantification of infarct size of different samples. $n=5$. (c) CK-MB, (d) LDH and (e) TNF- $\alpha$ levels of different samples were determined by commercial kits. $n=$ 8. Left ventricular function including (f) LVEDP, LVSP and $(\mathbf{g})+d P / d t$, $-d P / d t$. Results are expressed as mean $\pm S D, n=8$. $* P<0.05$, *** $P<0.0$ I versus $M I$ group. \#\# $P<0.0$ I versus sham group (one-way ANOVA, Dunnett test). Sham Sham operated; MImyocardial infarction; CPCardiotonic Pill; BECCs bioactive equivalent combinatorial components; MRCs mixture of reference compounds; Remaining part: CP lacking candidate BECCs.

assay, $P<0.0001$ for LDH assay, $n=8$, one-way ANOVA, Dunnett test). These results indicate that the cardioprotective effect of candidate BECGs (III) is comparable to that of CP in viwo.

MI resulted in a substantial increase of TNF- $\alpha$, while preincubation of CP or candidate BECGs (III) attenuated the elevation of TNF- $\alpha$ level (Fig. 5e, CP versus MI group, $P<0.0001$; candidate BECGs (III) versus MI group, $P<0.0001, n=8$, one-way ANOVA, Dunnett test). Compared with the sham group, LVEDP was increased while LVSP, maximal rate of increase of $L V$ pressure $(+d P / d t)$ and maximal rate of decrease of $L V$ pressure $(-d P / d t)$ were decreased postMI (Fig. 5f and g). Treatment with candidate BECGs (III) exhibited significant improvements on the heart's diastolic and systolic functions (Fig. $5 \mathrm{f}$ and g).

According to the bioactive equivalence assessment, candidate BECGs (III) suggested comparable efficacy with original CP within 90\% CI (Table III). The low activity of remaining part (III) also indicated that most of the bioactive components of CP were included in candidate BEGGs (III) (Fig. 5). Furthermore, MRGs (III) containing the same constituents as candidate 
Table III Bioactive Equivalence Evaluation of Candidate BECCs (III) and MRCs (III) in a Rat Model of Myocardial Infarction

\begin{tabular}{lcc}
\hline In vivo assay & Candidate BECCs (III) & \multicolumn{1}{l}{ MRCs (III) } \\
\hline Infarct size & $86 .|-| 15.9 \%$ & $80.2-109.2 \%$ \\
CK-MB & $\mid 31.8-153.0 \%$ & $104.2-135.9 \%$ \\
LDH & $\mid 17.2-248.8 \%$ & $\mid 10.5-188.4 \%$ \\
LVSP & $84.8-107.8 \%$ & $76.2-100.0 \%$ \\
LVEDP & $82.6-97.2 \%$ & $73.4-87.8 \%$ \\
$+d P / d t$ & $\mid 41.3-166.6 \%$ & $127.2-156.5 \%$ \\
$-d P / d t$ & $98.3-111.6 \%$ & $84.9-109.4 \%$ \\
TNF- $\alpha$ & $84.5-100.3 \%$ & $81.5-99.2 \%$ \\
\hline
\end{tabular}

$n=5-8$. Bioactive equivalence was evaluated by two one-sided $t$ test. The $90 \% \mathrm{Cl}$ of relative efficacy of candidate BECCs (III) and MRCs (III) compared to original CP was showd in the table. If the $90 \% \mathrm{Cl}$ of relative efficacy fell within the range of $70-143 \%$, the candidate BECCs were considered to be a bioactive equivalent with original Cardiotonic Pill

BECCs bioactive equivalent combinatorial components; MRCs mixture of reference compounds

BECCs (III) (Table S2, Supplementary Material) showed marked protective effects against MI (Fig. 5). Bioactive equivalence data were also consistent with that of candidate BECGs (III) (Table III).

The results of metabolomic profiling were given as PLSDA score plot that represents the metabolomics similarities of the samples (Fig. S5). The score plot of PLS-DA showed that both BECGs- and CP-treated groups were close to the sham group. It was demonstrated that the therapeutic efficacy of candidate BECCs (III) against MI is comparable to that of original CP.

\section{DISCUSSION}

Natural products and herbal medicines have played an important role in human health care and treatment of diseases for thousands of years $(1,2)$. Herbal medicines contain complex and relatively unrefined mixtures of compounds, the holistic efficacy of herbal medicines is usually a product of the unresolved integrative effects between the constituents (3-7). It is not surprising that the previous efforts in isolating and screening single compounds from herbal medicines are less satisfactory as expected because any single compound cannot stand for the whole therapeutic efficacies of herbal medicines. Thus scientific studies should examine the effect of multiple constituents taken together rather than testing single compounds one at a time. It is believed that not all the components contribute to the efficacy of herbal medicines, and some constituents contribute largely to the effects. We assumed that the exact composition of combinatorial components accounting for the whole efficacy of original herbal medicines can be refined from the complex mixtures, and defined as bioactive equivalent combinatorial components (BECGs).

To discover BECGs from herbal medicines of interest, we have established a feedback screening method, as illustrated in Fig. 6. The operations include chemical profiling of herbal medicines, followed by an iterative loop of determining, collecting and evaluating candidate BECGs.

To profile the chemical constituents in complex herbal medicines, diverse chromatographic methods can be used, such as high-performance liquid chromatography (HPLC) coupled with ultraviolet detection (UV), evaporative light

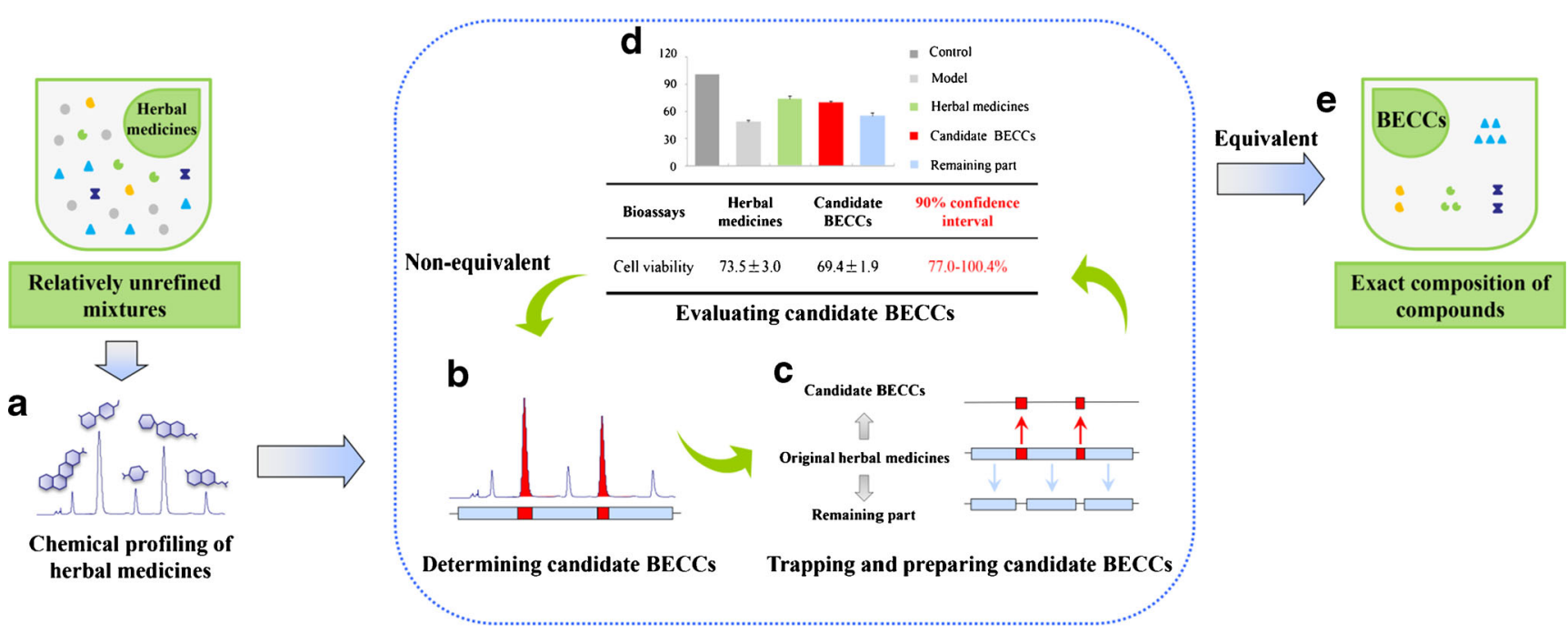

Fig. 6 A schematic illustration of bioactive equivalence oriented feedback screening method. The operations include chemical profiling of herbal medicines, followed by an iterative loop of determining, collecting and evaluating candidate BECCs. (a) Herbal medicines of interest are chemically profiled. (b) Candidate BECCs are determined from original herbal medicines according to a predefined selection criterion. (c) Candidate BECCs are trapped and prepared, the remaining part is obtained at the same time. (d) Evaluate the bioactivity of candidate BECCs and assess bioactive equivalence between candidate BECCs and original herbal medicines. If not bioactive equivalent, candidate BECCs will be reselected until they achieve bioactive equivalence with original herbal medicines. (e) The BECCs are identified which can account for the whole therapeutic efficacy of original herbal medicines. BECCs bioactive equivalent combinatorial components. 
scattering detector (ELSD) or mass spectrometry (MS). Furthermore, quadrupole time-of-flight mass spectrometry (QTOF MS) followed by database searching can provide structural information for unknown components.

The next step of the screening method is to determine which components in herbal medicines as temporally separated by liquid chromatography are candidates for inclusion in BECGs. In general, selection criteria for candidate BECGs are flexible. While normalized peak area ratio (\%) was chosen as the selection criterion in this work, identified chemical structure, chemical polarity, or binding affinity to bioactive macromolecules can all be considered as reference parameters (34-36). Indeed, any components of interest or their combinations can be selected and evaluated.

To obtain a combination of candidate BECCs based on a predefined criterion, we engineered a real-time components trapping and combining system (Figure S1, Supplementary Material). Candidate BECGs and the remaining parts of herbal medicines can be prepared at the same time and used for bioactivity evaluation. Importantly, the concentration and proportion of constituents in candidate BECGs remained the same as that in original herbal medicines for bioactivity assay. Because the preparation process may potentially lead to sample loss, the investigation of the preparative recovery and repeatability for candidate BECGs is essential to ensure the feasibility and reliability of the preparation system.

To address the issue of comparing efficacy of the candidate BECGs with the original herbal medicines, we propose a concept of bioactive equivalence that is similar to bioequivalence in pharmacokinetics (37). Candidate BECCs are considered to be bioactive equivalents with original herbal medicines if the ratio of their efficacies falls within an acceptable range for a given assay. In this study, the efficacies of candidate BECCs and original CP were evaluated in three cell models and a rat model of MI. According to the efficacy assessment, selection criteria were stepwise adjusted until the candidate BECCs could achieve bioactive equivalence with original herbal medicines.

A combination of 18 compounds was identified as BECGs from CP using the bioactive equivalence oriented feedback screening method. We suggest that the developed screening method and the discovered BECGs can be applied for the selection of chemical markers for quality control of herbal medicines. Chemical markers are key to ensure the efficacy, safety and batch-to-batch consistency of herbal medicines. In most cases, due to insufficient chemical and pharmacological data for herbal medicines, lack of evidence-based chemical markers remains a major challenge for the quality control of herbal medicines $(38,39)$. Using the described bioactive equivalence oriented feedback screening method, we can assess the rationality of designed chemical markers involved in Pharmacopeias and may suggest more suitable chemical markers. It is worth noting that the content of BECGs was $15.0 \%(w / w)$ of the original CP, and could be considered as the "defined labeled amount" of active constituents for CP against MI. It is promising that this screening method has a great potential in promoting standardization of herbal medicines. There are other possible applications of this method, for example in the assessment of toxic compounds or the analysis of integrative mechanisms of unrefined mixtures. The successful identification of naturally existing and integrative BECGs from the original efficacious formula leads us to suggest that our screening method could be used for multicomponent-based drug design $(40,41)$.

\section{CONCLUSION}

In conclusion, using the bioactive equivalence oriented feedback screening method, a combination of 18 compounds was identified as BECGs from Cardiotonic Pill (CP), which accounts for $15.0 \%(\mathrm{w} / \mathrm{w})$ of original CP. We have demonstrated that the BECGs were as effective as CP in cell culture experiments and in a rat model of MI. Our future studies will focus on optimization of the BECGs and the elucidation of their multiple targets and possible synergistic mechanisms.

\section{ACKNOWLEDGMENTS AND DISCLOSURES}

We gratefully acknowledge Prof. Jing Shang for her help on the in vitro studies and Dr. Jon Rees from Centers for Disease Control and Prevention in Atlanta for editing of the manuscript. This work was supported by the National Natural Science Foundation of China (Grant no. 81130068).

Open Access This article is distributed under the terms of the Creative Commons Attribution License which permits any use, distribution, and reproduction in any medium, provided the original author(s) and the source are credited.

\section{REFERENCES}

1. Cheung F. TCM: made in China. Nature. 2011;480(7378):S82-3.

2. Jiang WY. Therapeutic wisdom in traditional Chinese medicine: a perspective from modern science. Trends Pharmacol Sci. 2005;26(11):558-63.

3. Chen ST, Dou J, Temple R, Agarwal R, Wu KM, Walker S. New therapies from old medicines. Nat Biotechnol. 2008;26(10):1077-83.

4. Wang L, Zhou GB, Liu P, Song JH, Liang Y, Yan XJ, et al. Dissection of mechanisms of Chinese medicinal formula Realgar-Indigo naturalis as an effective treatment for promyelocytic leukemia. Proc Natl Acad Sci U S A. 2008;105(12):4826-31.

5. Schmidt BM, Ribnicky DM, Lipsky PE, Raskin I. Revisiting the ancient concept of botanical therapeutics. Nat Chem Biol. 2007;3(7):360-6. 
6. Ma XH, Zheng CJ, Han LY, Xie B, Jia J, Cao ZW, et al. Synergistic therapeutic actions of herbal ingredients and their mechanisms from molecular interaction and network perspectives. Drug Discov Today. 2009;14(11-12):579-88.

7. Williamson EW. Synergy and other interactions in phytomedicines. Phytomedicine. 2001;8(5):401-9.

8. Li JW, Vederas JC. Drug discovery and natural products: end of an era or an endless frontier? Science. 2009;325(5937):161-5.

9. Xu Z. Modernization: one step at a time. Nature. 2011;480(7378): S90-2.

10. Jia Y, Huang F, Zhang S, Leung SW. Is danshen (Salvia miltiorrhiza) dripping pill more effective than isosorbide dinitrate in treating angina pectoris? A systematic review of randomized controlled trials. Int J Cardiol. 2012;157(3):330-40.

11. Lu T, YangJ, Gao X, Chen P, Du F, Sun Y, et al. Plasma and urinary tanshinol from Salvia miltiorrhiza (Danshen) can be used as pharmacokinetic markers for cardiotonic pills, a cardiovascular herbal medicine. Drug Metab Dispos. 2008;36(8):1578-86.

12. Ma J, Qi LW, Li HJ, Li P. A segmental monitoring strategy based on variable wavelength detection for quality control of three Polygonaceae herbs. J Pharm Biomed Anal. 2012;62:155-61.

13. MacLellan WR, Wang Y, Lusis AJ. Systems-based approaches to cardiovascular disease. Nat Rev Cardiol. 2012;9(3):172-84.

14. Meilhac O, Zhou M, Santanam N, Parthasarathy S. Lipid peroxides induce expression of catalase in cultured vascular cells. J Lipid Res. 2000;41(8):1205-13.

15. Cominacini L, Pasini AF, Garbin U, Davoli A, Tosetti ML, Campagnola M, et al. Oxidized low density lipoprotein (ox-LDL) binding to ox-LDL receptor-1 in endothelial cells induces the activation of NF- $\mathrm{KB}$ through an increased production of intracellular reactive oxygen species. J Biol Chem. 2000;275(17):12633-8.

16. Chi YS, Cheon BS, Kim HP. Effect of wogonin, a plant flavone from Scutellaria radix, on the suppression of cyclooxygenase- 2 and the induction of inducible nitric oxide synthase in lipopolysaccharidetreated RAW 264.7 cells. Biochem Pharmacol. 2001;61(10):1195-203.

17. Bao Y, Lin C, Ren J, Liu J. MicroRNA-384-5p regulates ischemiainduced cardioprotection by targeting phosphatidylinositol-4,5bisphosphate3-kinase, catalytic subunit delta (PI3K pl108). Apoptosis. 2013;18(3):260-70.

18. Karalis V, Symillides M, Macheras P. Bioequivalence of highly variable drugs: a comparison of the newly proposed regulatory approaches by FDA and EMA. Pharm Res. 2012;29(4):1066-77.

19. Chen WL, Qian Y, Meng WF, Pang JY, Lin YC, Guan YY, et al. A novel marine compound xyloketal $\mathrm{B}$ protects against oxidized LDLinduced cell injury in vitro. Biochem Pharmacol. 2009;78(8):941-50.

20. Green LC, Wagner DA, Glogowski J, Skipper PL, Wishnok JS, Tannenbaum SR. Analysis of nitrate, nitrite, and $[15 \mathrm{~N}]$ nitrate in biological fluids. Anal Biochem. 1982;126(1):131-8.

21. Klocke R, Tian W, Kuhlmann MT, Nikol S. Surgical animal models of heart failure related to coronary heart disease. Cardiovasc Res. 2007;74(1):29-38.

22. Han SY, Li HX, Ma X, Zhang K, Ma ZZ, Jiang Y, et al. Evaluation of the anti-myocardial ischemia effect of individual and combined extracts of Panax notoginseng and Carthamus tinctorius in rats. J Ethnopharmacol. 2013;145(3):722-7.

23. Ytrehus K. The ischemic heart-experimental models. Pharmacol Res. 2000;42(3):193-203.

24. Johns TN, Olson BJ. Experimental myocardial infarction: I. a method of coronary occlusion in small animals. Ann Surg. 1954;140(5):675-82.
25. Hayakawa Y, Chandra M, Miao W, ShiraniJ, Brown JH, Dorn 2nd GW. Inhibition of cardiac myocyte apoptosis improves cardiac function and abolishes mortality in the peripartum cardiomyopathy of Gaq transgenic mice. Circulation. 2003;108(24):3036-41.

26. Chan D, Ng LL. Biomarkers in acute myocardial infarction. BMC Med. 2010;8:34.

27. Ridker PM, Rifai N, Pfeffer M, Sacks F, Lepage S, Braunwald E. Elevation of tumor necrosis factor- $\alpha$ and increased risk of recurrent coronary events after myocardial infarction. Circulation. 2000;101(18): 2149-53.

28. Pfeffer MA, Braunwald E. Ventricular remodeling after myocardial infarction. Experimental observations and clinical implications. Circulation. 1990;81(4):1161-72.

29. Moon C, Krawczyk M, Ahn D, Ahmet I, Paik D, Lakatta EG, et al. Erythropoietin reduces myocardial infarction and left ventricular functional decline after coronary artery ligation in rats. Proc Natl Acad Sci U S A. 2003;100(20):1 1612-7.

30. Liu L, Aa J, Wang G, Yan B, Zhang Y, Wang X, et al. Differences in metabolite profile between blood plasma and serum. Anal Biochem. 2010;406(2):105-12.

31. A J, Trygg J, Gullberg J, Johansson AI, Jonsson P, Antti H, et al. Extraction and GC/MS analysis of the human blood plasma metabolome. Anal Chem. 2005;77:8086-94.

32. Zhang H, Wang SQ Liu Y, Luo LP, Liu P, Qi LW, et al. Trace analysis in complex mixtures using a high-component filtering strategy with liquid chromatography-mass spectrometry. J Pharm Biomed Anal. 2012;70:169-77.

33. Qiao X, Zhang YT, Ye M, Wang BR, Han J, Guo DA, et al. Analysis of chemical constituents and taxonomic similarity of Salvia species in China using LC/MS. Planta Med. 2009;75(15):1613-7.

34. Chang KJ, Hazum E, Cuatrecasas P. Novel opiate binding sites selective for benzomorphan drugs. Proc Natl Acad Sci U S A. 1981;78(7):4141-5.

35. Zhou JL, An JJ, Li P, Li HJ, Jiang Y, Cheng JF. Two-dimensional turbulent flow chromatography coupled on-line to liquid chromatography-mass spectrometry for solution-based ligand screening against multiple proteins. J Chromatoqr A. 2009;1216(12):2394403.

36. Sun M, Ren J, Du H, Zhang Y, ZhangJ, Wang S, et al. A combined A431 cell membrane chromatography and online high performance liquid chromatography/mass spectrometry method for screening compounds from total alkaloid of Radix Caulophylli acting on the human EGFR. J Chromatogr B Analyt Technol Biomed Life Sci. 2010;878(28):2712-8.

37. Chen ML, Shah V, Patnaik R, Adams W, Hussain A, Conner D, et al. Bioavailability and bioequivalence: an FDA regulatory overview. Pharm Res. 2001;18(12):1645-50.

38. Xie P, Chen S, Liang YZ, Wang X, Tian R, Upton R. Chromatographic fingerprint analysis-a rational approach for quality assessment of traditional Chinese herbal medicine. J Chromatoqr A. 2006;1112(1-2):171-80.

39. Liang YZ, Xie P, Chau F. Chromatographic fingerprinting and related chemometric techniques for quality control of traditional Chinese medicines. J Sep Sci. 2010;33(3):410-21.

40. Zhang S, Yang X, Morris ME. Combined effects of multiple flavonoids on breast cancer resistance protein (ABCG2)-mediated transport. Pharm Res. 2004;21(7):1263-73.

41. Woodcock J, Griffin JP, Behrman RE. Development of novel combination therapies. N Engl J Med. 201 1;364(11):985-7. 